\title{
Poloidal flow generation in the dynamics of Rossby waves
}

\author{
Alexander M. Balk \\ Department of Mathematics, University of Utah, 155 South 1400 East, Salt Lake City, Utah 84112, USA
}

(Received 28 July 2019; published 16 December 2019)

\begin{abstract}
This paper considers the dynamics in the Charney-Hasegawa-Mima equation, basic to several different phenomena. In each of them, the generation of poloidal/zonal flow is important. The paper suggests a possibility to generate such flows (which can serve as transport barriers). Namely, one needs to create significant increments and decrements in the neighborhoods of some wave vectors $\mathbf{k}_{1}$ and $\mathbf{k}_{2}$ (respectively) such that (1) $R_{\mathbf{k}_{1}}<R_{\mathbf{k}_{2}}$, where $R_{\mathbf{k}}$ is the spectral density of the extra invariant kernel ( $I=\int R_{\mathbf{k}} E_{\mathbf{k}} d \mathbf{k}$ is the extra invariant, with $E_{\mathbf{k}}$ being the energy spectrum), (2) $\left|\mathbf{k}_{1}\right|<\left|\mathbf{k}_{2}\right|$, and (3) $\mathbf{k}_{1}+\mathbf{k}_{2}$ is a poloidal/zonal wave vector. These three conditions define a quite narrow region.
\end{abstract}

DOI: 10.1103/PhysRevResearch.1.033180

\section{INTRODUCTION}

The quasigeostrophic or Charney-Hasegawa-Mima (CHM) equation $[1,2]$

$$
(1-\Delta) \psi_{t}+\psi_{y}=\psi_{x} \Delta \psi_{y}-\psi_{y} \Delta \psi_{x}
$$

is a basic model for several different phenomena, in particular, (i) ocean dynamics [3], (ii) tokamak plasmas [4,5], and (iii) slow magnetohydrodynamics in the ocean of the core [6] (in the latter case, instead of being the stream function, $\psi$ is the vertical component of the vector potential, so $-\psi_{y}, \psi_{x}$ are the horizontal components of the magnetic field) [7].

In all these situations the generation of poloidal/zonal flow is important. In the first case, emerging zonal flow limits the meridional transport. In the second case, poloidal flow limits the transport in the radial direction of a tokamak. In the third case, instead of zonal flow, we have the generation of a zonal magnetic field, important for dynamo theory.

Unlike cases (i) and (iii), in case (ii) we can actually change something, and this is the reason why the CHM equation is written here in plasma notations (with radial $x$ and poloidal $y$ coordinates; the units are chosen to make the coefficients equal 1).

The present paper describes what increments or decrements we could add to the CHM equation in order to generate or to aid in the generation of poloidal flow (a transport barrier).

In the Fourier representation, Eq. (1) — with the increments $\gamma_{\mathbf{k}}$-becomes

$$
\dot{\psi}_{\mathbf{k}}+i \Omega_{\mathbf{k}} \psi_{\mathbf{k}}=\frac{1}{2} \int W_{-\mathbf{k}, \alpha, \beta} \psi_{\alpha} \psi_{\beta} d_{\alpha \beta}+\gamma_{\mathbf{k}} \psi_{\mathbf{k}},
$$

Published by the American Physical Society under the terms of the Creative Commons Attribution 4.0 International license. Further distribution of this work must maintain attribution to the author(s) and the published article's title, journal citation, and DOI. where indices $\alpha, \beta$ (and later $\lambda, \mu, v$ ) stand for the corresponding wave vectors $\left(\mathbf{k}_{\alpha}, \ldots\right) . d_{\alpha \beta}=d \mathbf{k}_{\alpha} d \mathbf{k}_{\beta}$. Here,

$$
\Omega_{\mathbf{k}}=\frac{q}{1+k^{2}}
$$

is the dispersion law [wave vector $\mathbf{k}=(p, q), k^{2}=p^{2}+q^{2}$ ]; the coupling kernel $W_{-\mathbf{k} \alpha \beta}=U_{-\mathbf{k} \alpha \beta} \delta\left(-\mathbf{k}+\mathbf{k}_{\alpha}+\mathbf{k}_{\beta}\right)$,

$$
U_{-\mathbf{k} \alpha \beta}=\left(p_{\alpha} q_{\beta}-p_{\beta} q_{\alpha}\right) \frac{k_{\beta}^{2}-k_{\alpha}^{2}}{1+k^{2}} .
$$

\section{EXTRA INVARIANT}

Equation (1) is remarkable in the following sense [8,9]. In addition to the energy and the momentum (the enstrophy is their linear combination), this equation has an (independent) extra invariant conserved adiabatically, i.e., approximately over a long time [10]. To see this, consider the quantity

$$
I=\frac{1}{2} \int X_{\mathbf{k}}\left|\psi_{\mathbf{k}}\right|^{2} d \mathbf{k}+\frac{1}{6} \int Y_{\lambda \mu \nu} \psi_{\lambda} \psi_{\mu} \psi_{\nu} d_{\lambda \mu \nu},
$$

with undetermined coefficient functions $X$ and $Y$ (without loss of generality, $Y$ is independent of the order of its indices, and $X$ is even, $X_{\mathbf{k}}=X_{-\mathbf{k}}$ since $\left.\overline{\psi_{\mathbf{k}}}=\psi_{-\mathbf{k}}\right)$. The time derivative of (5) due to Eq. (2) is

$$
\begin{aligned}
\dot{I}= & \int \gamma_{\mathbf{k}} X_{\mathbf{k}}\left|\psi_{\mathbf{k}}\right|^{2} d \mathbf{k} \\
& +\frac{1}{6} \int\left\{X_{\lambda} W_{\lambda \mu \nu}+X_{\mu} W_{\mu \nu \lambda}+X_{\nu} W_{\nu \lambda \mu}\right. \\
& \left.+\left(\gamma_{\lambda}-i \Omega_{\lambda}+\gamma_{\mu}-i \Omega_{\mu}+\gamma_{\nu}-i \Omega_{v}\right) Y_{\lambda \mu \nu}\right\} \psi_{\lambda} \psi_{\mu} \psi_{\nu} d_{\lambda \mu \nu} \\
& +\frac{1}{4} \int W_{-\lambda \alpha \beta} Y_{\lambda \mu \nu} \psi_{\alpha} \psi_{\beta} \psi_{\mu} \psi_{\nu} d_{\alpha \beta \mu \nu} .
\end{aligned}
$$

Suppose the drift waves have small amplitudes and small increments: $\psi_{\mathbf{k}}=O(\epsilon)$ and $\gamma_{\mathbf{k}}=O(\epsilon)$, where $\epsilon$ is a small parameter. Cubic $O\left(\epsilon^{3}\right)$ terms in Eq. (6) would cancel if

$$
Y_{\lambda \mu \nu}=\frac{X_{\lambda} W_{\lambda \mu \nu}+X_{\mu} W_{\mu \nu \lambda}+X_{\nu} W_{\nu \lambda \mu}}{i\left(\Omega_{\lambda}+\Omega_{\mu}+\Omega_{v}\right)},
$$




$$
\Longrightarrow \quad \dot{I}=\int \gamma_{\mathbf{k}} X_{\mathbf{k}}\left|\psi_{\mathbf{k}}\right|^{2} d \mathbf{k}+O\left(\epsilon^{4}\right) .
$$

Without an increment $\left(\gamma_{\mathbf{k}} \equiv 0\right)$, we have $\dot{I}=O\left(\epsilon^{4}\right)$, while $I=O\left(\epsilon^{2}\right)$; integrating in time, we find $\Delta I \equiv I(t)-I(0)=$ $O\left(\epsilon^{3}\right)$ over a long time $t=O\left(\epsilon^{-1}\right)$. Notice that the $Y$ term in Eq. (5) has the same order $O\left(\epsilon^{3}\right)$ as $\Delta I$, and so the $Y$ term can be eventually discarded.

The above argument tacitly assumes that expression (7) does not blow up when its denominator vanishes. The latter condition turns out to be very restrictive [11], realizable only for some special functions $X_{\mathbf{k}}$ : If $X_{\mathbf{k}}=1+k^{2}$, then $Y \equiv 0$, and $I$ is the energy. If $X_{\mathbf{k}}=k^{2}\left(1+k^{2}\right)$, then $Y \equiv 0$, and $I$ is the enstrophy.

There is one choice with nonzero $Y: X_{\mathbf{k}}=\frac{\left(1+k^{2}\right)^{2}}{q} \eta_{\mathbf{k}}$,

$$
\eta_{\mathbf{k}}=\arctan \left(\frac{p+\sqrt{3} q}{k^{2}}\right)-\arctan \left(\frac{p-\sqrt{3} q}{k^{2}}\right) .
$$

The requirement of no blowup in Eq. (7) is reduced [12] to the conservation of function (9) in the three-wave resonance interactions:

$$
\left.\begin{array}{c}
\mathbf{k}_{\lambda}+\mathbf{k}_{\mu}+\mathbf{k}_{v}=\mathbf{0}, \\
\Omega_{\lambda}+\Omega_{\mu}+\Omega_{v}=0
\end{array}\right\} \Rightarrow \eta_{\lambda}+\eta_{\mu}+\eta_{v}=0
$$

[recall that all three $W$ kernels in Eq. (7) contain the same delta function $\delta\left(\mathbf{k}_{\lambda}+\mathbf{k}_{\mu}+\mathbf{k}_{v}\right)$ ]. Condition (10) uniquely [13] determines function (9)—up to linear combinations: Obviously, any linear combination of functions $\mathbf{k}, \Omega_{\mathbf{k}}, \eta_{\mathbf{k}}$ is also conserved in the three-wave resonance interactions. Actually, it is beneficial, instead of function $\eta_{\mathbf{k}}$, to consider function $\tilde{\eta}_{\mathbf{k}}=\eta_{\mathbf{k}}-2 \sqrt{3} \Omega_{\mathbf{k}}$. This combination vanishes as $k \rightarrow \infty$, faster than $\eta_{\mathbf{k}}$ and $\Omega_{\mathbf{k}}$ separately, and this takes place along all directions in the $\mathbf{k}$ plane. The function $\tilde{\eta}_{\mathbf{k}}$ gives a well-defined invariant that holds in the physical space as well [14]. The combination $\tilde{\eta}_{\mathbf{k}}$ also vanishes faster than $\eta_{\mathbf{k}}$ and $\Omega_{\mathbf{k}}$ separately, as $q \rightarrow 0$, for any $p$.

Thus, Eq. (1) has three invariants:

$$
\text { energy: } E=\frac{1}{2} \int E_{\mathbf{k}} d \mathbf{k}, \quad \dot{E}=\int \gamma_{\mathbf{k}} E_{\mathbf{k}} d \mathbf{k},
$$$$
\text { enstrophy: } \Phi=\frac{1}{2} \int k^{2} E_{\mathbf{k}} d \mathbf{k}, \quad \dot{\Phi}=\int \gamma_{\mathbf{k}} k^{2} E_{\mathbf{k}} d \mathbf{k} \text {, }
$$

$$
\text { extra invariant: } I=\frac{1}{2} \int R_{\mathbf{k}} E_{\mathbf{k}} d \mathbf{k}, \quad \dot{I}=\int \gamma_{\mathbf{k}} R_{\mathbf{k}} E_{\mathbf{k}} d \mathbf{k},
$$

$$
R_{\mathbf{k}}=\frac{\eta_{\mathbf{k}}-2 \sqrt{3} \Omega_{\mathbf{k}}}{\Omega_{\mathbf{k}}} .
$$

We should keep in mind that the extra conservation holds only for weak nonlinearity, when $\epsilon$ is small enough, and the reminder $O\left(\epsilon^{4}\right)$ in Eq. (8) can be neglected.

All three invariants (11) are positive-definite.

\section{ENERGY TRANSFER}

The presence of the extra invariant leads to an essential conclusion about the energy transfer from the source (acting at some scale) to other scales [8]. The following argument [15] describes the emergence of poloidal flow.

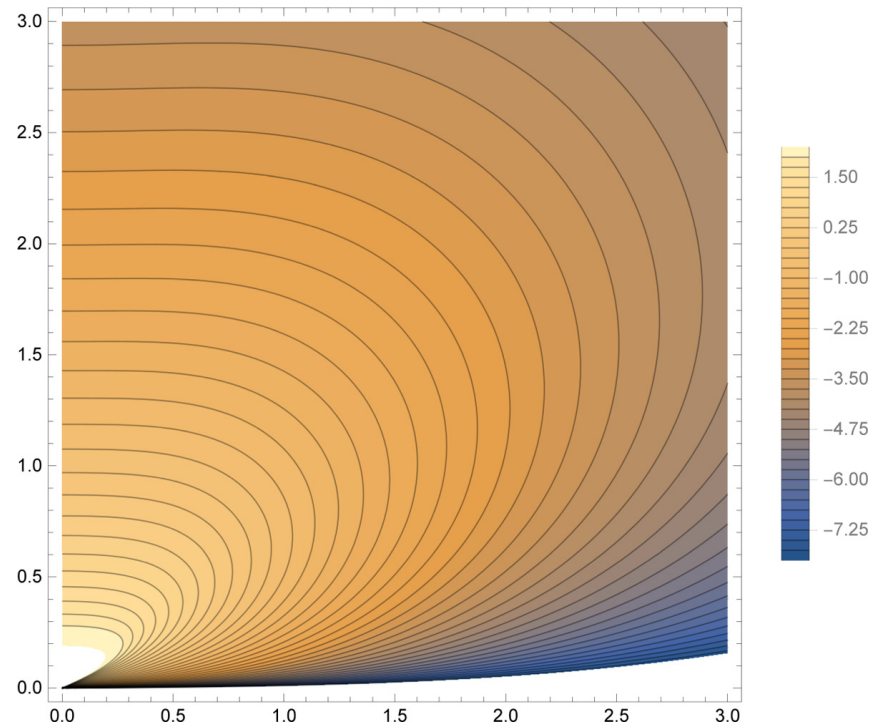

FIG. 1. Contour plot of the ratio (12). The values of the color bar are proportional to $\log _{10} R_{\mathbf{k}}$. The white spots at the bottom correspond to the values of $\log _{10} R_{\mathrm{k}}$ out of the range of the color bar $\left(R \sim \pi / q \rightarrow \infty\right.$ as $k \rightarrow 0,|p / q|<\sqrt{3}$, and $R \propto q^{2} \rightarrow 0$ as $\left.q \rightarrow 0\right)$.

Due to the enstrophy conservation, the energy from the source would transfer towards larger scales, i.e., towards the origin in the $k$ plane. Due to the extra conservation, the energy should concentrate near the $p$ axis, that corresponds to the poloidal flow.

Indeed, Fig. 1 shows the contour plot of the ratio (12). We see that $R_{\mathbf{k}}$ decreases when $k$ becomes larger or when $q$ becomes smaller; more precisely,

$$
R_{\mathbf{k}}=8 \sqrt{3} \times \begin{cases}\frac{5 p^{2} q^{2}+q^{4}}{5 k^{8}}+O\left(k^{-6}\right), & k \rightarrow \infty \\ \frac{q^{2}}{p^{2}\left(1+p^{2}\right)^{2}}+O\left(q^{4}\right), & q \rightarrow 0 .\end{cases}
$$

So, if the energy from the source were transferred away from the $p$ axis, then the extra invariant would significantly increase: From the right upper corner (big $k$ ) in Fig. 1 to the left bottom corner (small $k$ ) the ratio $R$ changes by nine orders of magnitude, provided the small wave vectors belong to the sector of polar angles $\theta \equiv \arctan (q / p)>30^{\circ}$. When $\theta$ decreases from $30^{\circ}$ to $0^{\circ}$, the ratio $R$ decreases to zero. So, the difference in scales (between the source scale and the big scale) should be large enough to ensure the energy concentration near the $p$ axis.

Thus, the poloidal flow is always generated (without us doing anything), provided the scales are disparate enough. This reasoning equally applies to the cascade (local in the $\mathbf{k}$ plane) or nonlocal energy transfer.

\section{ENERGY TRANSFER WITHOUT SCALE DISPARITY}

However, there is a problem with the above argument: It is hardly possible in practice that the drift waves are weakly nonlinear in a wide range of scales. Usually, smaller scales are strongly nonlinear, while larger scales are weakly nonlinear (e.g., Ref. [16]). The present paper is motivated by two goals. First, we will see the possibility of zonal flow generation without the presence of a wide range of scales, namely, 
without small scales. However, for this, we do need to arrange increments and decrements in a certain special way. The large scales do have to be weakly nonlinear, but this matches the stability of zonal waves in the weakly nonlinear limit (see below).

The second goal is to make a specific suggestion about how one could generate poloidal (zonal) flow. This suggestion is based on the fact-not noticed earlier - that the contour lines in Fig. 1 have a "depression" in some region near the $q$ axis (away from the poloidal flow, corresponding to the $p$ axis): In this region, the ratio $R_{\mathbf{k}}$ is an increasing function of $p$ (while $q$ is fixed). This "depression" is hardly visible in Fig. 1.

Let there be a positive increment in a small neighborhood $B_{1}$ of some wave vector $\mathbf{k}_{1}$ and a decrement in a small neighborhood $B_{2}$ of some $\mathbf{k}_{2}$, while $\gamma_{\mathbf{k}} \equiv 0$ everywhere else. Consider the positive quantities

$$
G_{1}=\int_{0}^{\infty} d t \int_{B_{1}} \gamma_{\mathbf{k}} E_{\mathbf{k}} d \mathbf{k}, \quad G_{2}=-\int_{0}^{\infty} d t \int_{B_{2}} \gamma_{\mathbf{k}} E_{\mathbf{k}} d \mathbf{k} .
$$

According to (11),

$$
\begin{aligned}
E^{\star} & =E^{0}+G_{1}-G_{2}, \\
\Phi^{\star} & =\Phi^{0}+k_{1}^{2} G_{1}-k_{2}^{2} G_{2}, \\
I^{\star} & =I^{0}+R_{1} G_{1}-R_{2} G_{2},
\end{aligned}
$$

where $E^{0}, \Phi^{0}, I^{0}$ are the initial values (at $t=0$ ) of the energy, enstrophy, and extra invariant; their final values (at $t=\infty$ ) are $E^{\star}, \Phi^{\star}, I^{\star} ; R_{j}=R_{\mathbf{k}_{j}}(j=1,2)$. A slight difference between $k_{1}$ and $k_{2}$ (respectively, $R_{1}$ and $R_{2}$ ) could produce a large difference between $\Phi^{0}$ and $\Phi^{\star}\left(I^{0}\right.$ and $\left.I^{\star}\right)$ if the time interval is long enough.

We want to generate a significant amount of energy $\left(G_{1}>\right.$ $G_{2}$ ) and to have a small amount of the extra invariant $I^{\star} \approx$ 0 . (Recall that the vanishing of the extra invariant requires vanishing of all drift waves besides the poloidal flow.) If $I^{\star}<I^{0}$, i.e., $R_{1} G_{1}-R_{2} G_{2}<0$, then

$$
R_{1}<R_{2} \text {. }
$$

Condition (14) is implied by even less stringent requirements that the generated extra invariant per generated energy $\frac{R_{1} G_{1}-R_{2} G_{2}}{G_{1}-G_{2}}$ is less than $R_{1}$ or $R_{2}$.

We want to generate large-scale flow. In other words, the energy should concentrate in longer waves. The latter carry less enstrophy per energy than shorter waves. Thus, we should pump mostly energy and dissipate mostly enstrophy, i.e.,

$$
k_{1}<k_{2} \text {. }
$$

Formally, condition (15) follows from the requirement that the generated enstrophy per generated energy $\frac{k_{1}^{2} G_{1}-k_{2}^{2} G_{2}}{G_{1}-G_{2}}$ is less than $k_{1}^{2}$ or $k_{2}^{2}$.

If (15) does not hold, $k_{1}>k_{2}$, then the generated enstrophy per generated energy could take any large values. Also, if $k_{1}>$ $k_{2}$, it is possible that the extra invariant becomes small, but the poloidal flow is not generated; this can happen because the ratio $R_{\mathrm{k}}$ quickly decreases as $k$ increases [see Fig. 1 and asymptotics (13)].

We want to generate poloidal flow, and so we require $\mathbf{k}_{3}=$ $\mathbf{k}_{1}+\mathbf{k}_{2}$ be a purely polodal wave vector:

$$
p_{2}=p_{3}-p_{1}, \quad q_{2}=-q_{1} .
$$

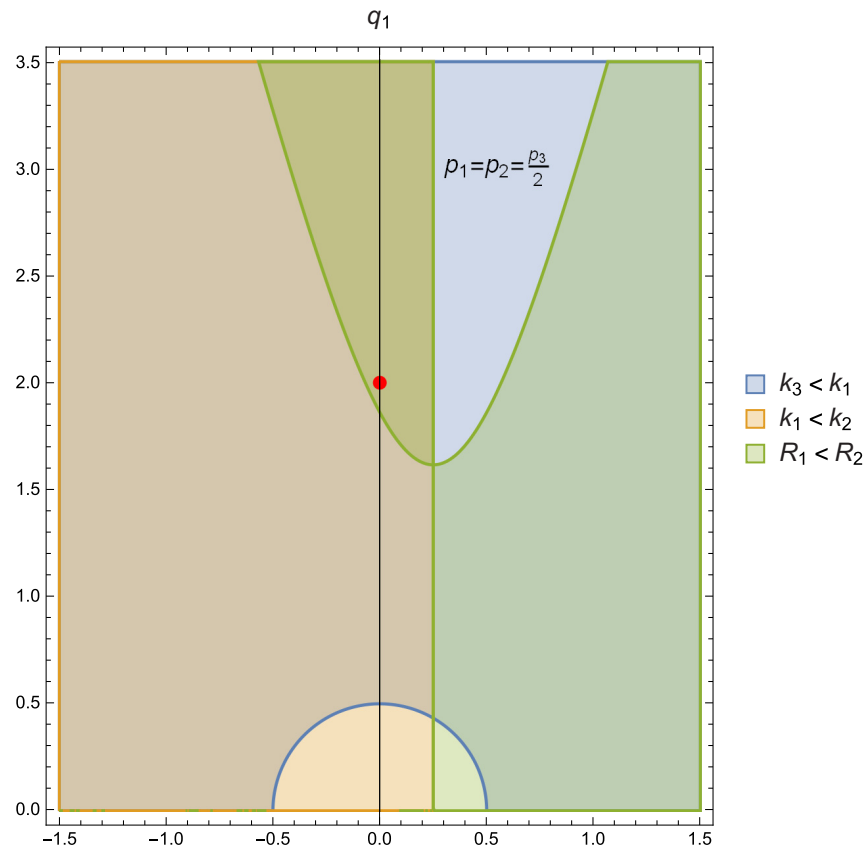

FIG. 2. Three regions in the $\mathbf{k}_{1}$ plane (while $p_{3}=1 / 2$ ): (i) Outside of the semicircle is the region $k_{3}<k_{1}$. (ii) To the left of the vertical line $p_{1}=p_{2}=p_{3} / 2$ is the region $k_{1}<k_{2}$. (iii) The region $R_{1}<R_{2}$ consists of two pieces: to the right of the line and outside of the parabolalike curve, and to the left of the line and inside the parabolalike curve. The intersection of regions (ii) and (iii) is the region satisfying conditions (14)-(16). Inside this intersection is the point $\mathbf{k}_{1}=(0,2)$ marked by the dot. (Due to the symmetry, only half of the domain, with $q_{1}>0$, is shown.)

Figure 2 shows region of the $\mathbf{k}_{1}$ plane determined by conditions (14)-(16), while $p_{3}$ is held fixed. These conditions automatically imply $k_{3}<k_{1}$. So, the wave $\mathbf{k}_{1}$-pumped due to the positive increment-can decay into the waves $\mathbf{k}_{2}$ and $\mathbf{k}_{3}$ [17].

\section{MODEL SIMULATIONS}

There is an additional bonus of condition (15): It implies that one can make a numerical simulation with a only few modes, as significantly shorter waves are not generated. The generation of much longer waves is also impossible if the scales corresponding to $k_{1}, k_{2}, k_{3}$ are close to the size of the system.

With this in mind, we consider the dynamics of only three waves [18] with wave vectors $\mathbf{k}_{3}=(1 / 2,0)$, $\mathbf{k}_{1}=(0,2)$ corresponding to the dot in Fig. 2, and $\mathbf{k}_{2}=\mathbf{k}_{3}-\mathbf{k}_{1}$,

$$
\begin{aligned}
& \dot{\psi}_{1}+i \Omega_{1} \psi_{1}=U_{1} \psi_{3} \overline{\psi_{2}}+\gamma_{1} \psi_{1}, \\
& \dot{\psi}_{2}+i \Omega_{2} \psi_{2}=U_{2} \psi_{3} \overline{\psi_{1}}+\gamma_{2} \psi_{2}, \\
& \dot{\psi}_{3}+i \Omega_{3} \psi_{3}=U_{3} \psi_{1} \psi_{2} ;
\end{aligned}
$$

$U_{1}=U_{\mathbf{k}_{1} \mathbf{k}_{3}-\mathbf{k}_{2}}, \quad U_{2}=U_{\mathbf{k}_{2} \mathbf{k}_{3}-\mathbf{k}_{1}}, \quad U_{3}=U_{\mathbf{k}_{3} \mathbf{k}_{1} \mathbf{k}_{2}}, \quad \Omega_{j}=\Omega_{\mathbf{k}_{j}}$ $(j=1,2,3), \gamma_{1}>0, \gamma_{2}<0$. 


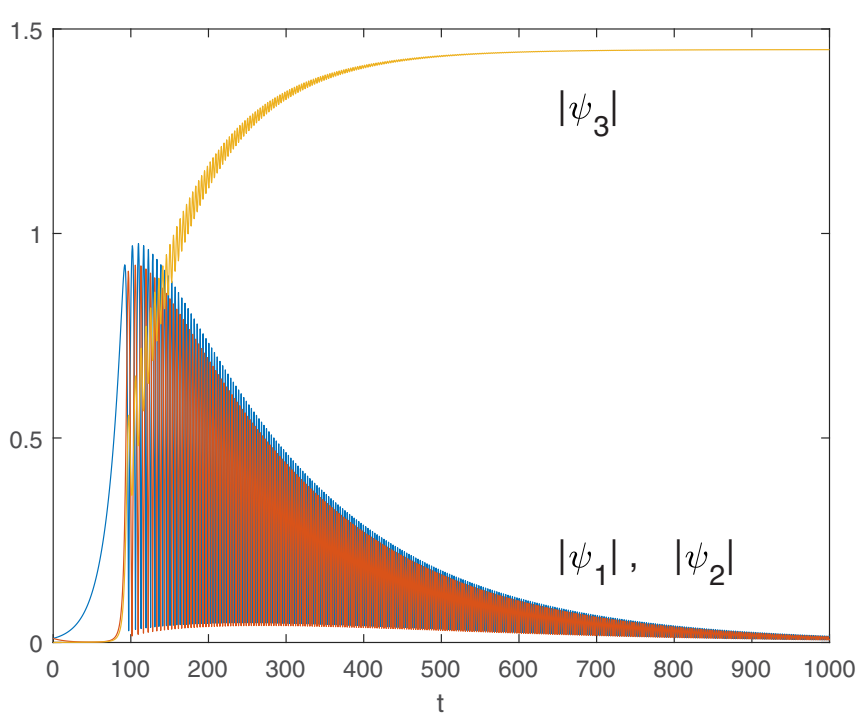

FIG. 3. Triad simulation. The curves appear having some width; this is due to oscillations (cf. Ref. [18]). (Note the figure shows oscillations of absolute values $|\psi|$, not $\psi$ themselves.) For this particular graph, $\gamma_{1}=0.05, \gamma_{2}=-0.06$, and $\left|\psi_{1}(0)\right|=\left|\psi_{2}(0)\right|=$ 0.01 , while $\left|\psi_{3}(0)\right|=0$ (the initial phases $\arg [\psi(0)]$ are random and appear insignificant).

There is long history of modeling fusion plasmas by various small systems of ordinary differential equations (ODE) (see Refs. [19,20] and references cited therein).

Figure 3 shows the emergence of poloidal flow in the model (17). I performed simulations using MATLAB ODE solvers with decreased absolute and relative tolerances (AbsTol and RelTol). Instead of MATLAB default values AbsTol $=10^{-6}$ and RelTol $=10^{-3}$, I used AbsTol $=10^{-13}$ and RelTol $=$ $10^{-11}$. These are needed for the validity of long-time simulations. I also checked that different ODE solvers produced indistinguishable results.

The relevance of these calculations to tokamak plasmas is due to the smallness in seconds of the timescale for drift waves. In particular, for ITER, the drift velocity is $v_{d} \sim$ $2 \mathrm{~km} / \mathrm{s}$ and the ion inertial (Rossby) radius $\rho \sim 3 \mathrm{~mm}$ [21]; therefore, the timescale is $\rho / v_{d} \sim(3 / 2) \times 10^{-6} \mathrm{~s}$. So, the dimensionless time $t=1000$ (the time range in Fig. 3) corresponds to $1.5 \times 10^{-3} \mathrm{~s}$; within a fraction of this time, the amplitude of the poloidal mode becomes significantly bigger than the amplitudes of the other two modes. The emergence of the poloidal flow would occur faster if the increment and decrement had bigger magnitudes.

\section{STABILITY CONSIDERATIONS AND CONCLUSION}

If the emerging flow were not poloidal, it would be unstable with respect to decays into other waves. But poloidal flow is stable in the weak interaction limit [17]. One can derive that the poloidal mode is stable if

$$
\left|p_{3} \psi_{3}\right|<1 /\left(1+k_{3}^{2}\right)
$$

Indeed, in the weak interaction limit, the instability of a Rossby wave $\mathbf{k}_{3}$ is the instability with respect to decays into a pair of waves, say $\mathbf{k}_{4}$ and $\mathbf{k}_{5}$, such that $\mathbf{k}_{3}=\mathbf{k}_{4}+\mathbf{k}_{5}$, and $k_{3}$ is in between $k_{4}$ and $k_{5}$ [17]. It is well known that this instability does not take place if

$$
D \equiv \omega^{2} / 4-U_{4} U_{5}\left|\psi_{3}\right|^{2}>0,
$$

where $\omega=\Omega_{4}+\Omega_{5}\left(\Omega_{3}=0\right)$, and according to (4),

$$
\begin{aligned}
& U_{4}=U_{\mathbf{k}_{4} \mathbf{k}_{3}-\mathbf{k}_{5}}=p_{3} q_{5}\left(k_{3}^{2}-k_{5}^{2}\right) /\left(1+k_{4}^{2}\right), \\
& U_{5}=U_{\mathbf{k}_{5} \mathbf{k}_{3}-\mathbf{k}_{4}}=p_{3} q_{4}\left(k_{3}^{2}-k_{4}^{2}\right) /\left(1+k_{5}^{2}\right) .
\end{aligned}
$$

Since $q_{4}+q_{5}=0$, we can write

$$
\omega=q_{4}\left(\frac{1}{1+k_{4}^{2}}-\frac{1}{1+k_{3}^{2}}\right)+q_{5}\left(\frac{1}{1+k_{5}^{2}}-\frac{1}{1+k_{3}^{2}}\right) .
$$

By the inequality for arithmetic and geometric means,

$$
\begin{aligned}
\left(\frac{\omega}{2}\right)^{2} & \geqslant q_{4} q_{5} \frac{\left(k_{3}^{2}-k_{4}^{2}\right)\left(k_{3}^{2}-k_{5}^{2}\right)}{\left(1+k_{4}^{2}\right)\left(1+k_{5}^{2}\right)\left(1+k_{3}^{2}\right)^{2}}, \Longrightarrow \\
D & \geqslant q_{4}^{2} \frac{\left(k_{4}^{2}-k_{3}^{2}\right)\left(k_{3}^{2}-k_{5}^{2}\right)}{\left(1+k_{4}^{2}\right)\left(1+k_{5}^{2}\right)}\left\{\frac{1}{\left(1+k_{3}^{2}\right)^{2}}-p_{3}^{2}\left|\psi_{3}\right|^{2}\right\} .
\end{aligned}
$$

So, $D>0$ if the condition (18) holds. The condition (18) says that the fluid velocity $v_{3}=i p_{3} \psi_{3}$, due to the poloidal wave, has a smaller magnitude than the velocity of this wave (its phase and group velocities coincide). This stability in the weak interaction limit matches the argument based on the extra invariant that requires weak nonlinearity as well.

To summarize, this paper suggests a specific possibility to generate poloidal flow, which can help in plasma confinement. Namely, the paper suggests a certain arrangement of increments and decrements, so that the energy is accumulated, while the extra invariant is "drained," and the amount of enstrophy per energy does not increase. The suggested possibility needs to be verified. The considered example [system (17), Fig. 3] illustrates this possibility, but clearly is insufficient for its verification.

The considerations in the present paper can be extended beyond the CHM equation (1) (e.g., Ref. [22]); the system should contain waves with dispersion law (3) and possess a Hamiltonian structure.
[1] J. G. Charney, The Atmosphere-A Challenge (Springer, 1990), pp. 251-265.

[2] A. Hasegawa and K. Mima, Phys. Rev. Lett. 39, 205 (1977).
[3] G. K. Vallis, Atmospheric and Oceanic Fluid Dynamics. Fundamentals and Large-Scale Circulation (Cambridge University Press, Cambridge, UK, 2006). 
[4] P. H. Diamond, S.-I. Itoh, and K. Itoh, Modern Plasma Physics (Cambridge University Press, Cambridge, UK, 2010), Vol. 1.

[5] W. Horton, Turbulent Transport in Magnetized Plasmas (World Scientific, Singapore, 2012).

[6] S. I. Braginsky, Earth Planet. Sci. Lett. 253, 507 (2007).

[7] A. M. Balk, Astrophys. J. 796, 143 (2014).

[8] A. M. Balk, S. V. Nazarenko, and V. E. Zakharov, Phys. Lett. A 152, 276 (1991).

[9] A. M. Balk, Phys. Lett. A 155, 20 (1991).

[10] Equation (1) has an infinite family of invariant Casimirs, but these explicitly depend on the coordinate $x$. Besides, if the equation is Fourier truncated, the Casimirs fail to be conservedunlike the energy, enstrophy, and extra invariant. This is due to the quadratic nature of the latter three invariants.

[11] V. E. Zakharov and E. I. Shul'man, Physica D 1, 192 (1980).

[12] A. M. Balk and F. van Heerden, Physica D 223, 109 (2006).
[13] A. M. Balk and E. V. Ferapontov, in Nonlinear Waves and Weak Turbulence, edited by V. E. Zakharov (Amer. Math. Soc. Trans. Ser. 2, 1998), Vol. 182, pp. 1-30.

[14] A. M. Balk and T. Yoshikawa, Physica D 238, 384 (2009).

[15] A. M. Balk, Phys. Lett. A 345, 154 (2005).

[16] P. B. Rhines, J. Fluid Mech. 69, 417 (1975).

[17] A. Gill, Geophys. Fluid Dyn. 6, 29 (1974).

[18] A. M. Balk, Phys. Rev. E 98, 062208 (2018).

[19] F. A. Marcus, M. Roberto, I. L. Caldas, K. C. Rosalem, and Y. Elskens, Phys. Plasmas 26, 022302 (2019).

[20] I. I. Rypina, M. G. Brown, F. J. Beron-Vera, H. Kocak, M. J. Olascoaga, and I. A. Udovydchenkov, Phys. Rev. Lett. 98, 104102 (2007).

[21] W. Horton and S. Benkadda, ITER Physics (World Scientific, Singapore, 2015).

[22] D. Qi and A. J. Majda, arXiv:1901.08590. 